\title{
REAL-TIME DECISION SUPPORT SYSTEM FOR CARBON MONOXIDE THREAT WARNING USING ONLINE EXPERT SYSTEM
}

\author{
Suryono Suryono ${ }^{1 *}$, Bayu Surarso ${ }^{1}$, Ragil Saputra ${ }^{1}$, Sudalma Sudalma ${ }^{2}$ \\ 'Diponegoro University, Semarang, Indonesia \\ ${ }^{2}$ Semarang Occupational Health and Safety Office, Indonesia
}

\begin{abstract}
This paper describes a decision support system to mitigate the danger that carbon monoxide pose via internet-based measurement. This system is required because in high concentration above threshold, carbon monoxide can trigger many diseases and even cause death. However, a system that is capable of detection and making online decision in real-time against that threat is not yet available. Therefore, decisions on carbon monoxide threat are often taken too late as they are made manually with expert analysis. This research proposes the design of a sensor node composed of a gas sensor, a microcontroller, a WIFI router, and an Internet modem to acquire data and communicate them via the internet. The pollution index value and rule-based algorithm, which are used to determine carbon monoxide gas pollution categories in the web server program, are in accordance to data stated in the Indonesia Air Pollutant Index. An expert system programming based on expert knowledge is then used to make decision on pollution. Results show that the sensor node built is capable of sending data online to the cloud station, with Root Mean Square Error of $3.46 \mu \mathrm{g} / \mathrm{m}^{2}$ and relative error of $0.78 \%$ for a measurement range of $0-440 \mu \mathrm{g} / \mathrm{m}^{2}$. This system is also capable of sending data fast with a transfer rate of 764 milliseconds. Further testing also revealed that using expert system in cloud computing results in speedy warning of carbon monoxide threat, at 15.6 seconds.
\end{abstract}

Key words: Internet-based, Carbon monoxide, Sensor node, Rule-based, Expert knowledge

\section{INTRODUCTION}

Monitoring of air quality is very important as it affects both health and work safety. The level of carbon monoxide (CO) in the environment is one of the indicators for air quality. $\mathrm{CO}$ is colorless and does not smell that it is hard to detect. At high concentration, CO is highly toxic and detrimental to health [01]. $C O$ results from burning of fossil fuels by vehicles, industries, and open burnings $[02,03]$. CO gets into the blood stream via the lungs and it ties with hemoglobin in the blood and is taken by the Oxygen $\left(\mathrm{O}_{2}\right)$ to cells.

Healthy people who are exposed to high level of $\mathrm{CO}$ are at risk of reduced mental alertness [04]. Real-time monitoring of $\mathrm{CO}$ level is important in order to measure air quality, because high concentration of $\mathrm{CO}$ is hard to detect by the human body [05]. Moreover, it is easily dispersed in open air. The width of coverage area is also another challenge for manual measurement. Furthermore, wireless sensor technology has many merits including low cost, minimum maintenance, and wide coverage area [06].

Wireless sensor allows measurement of environmental physical parameters in real-time [07]. Environmental monitoring requires the use of wireless sensor technology as the measurement is in the order of months and even years. Wireless sensor system allows data compression and prediction algorithm. This makes the system built is of high efficiency [08]. Combining wireless sensor system with web based online technology enables integration of sensors into a system that can be remotely accessed in real-time. The use of this system also helps to ease data communication as to improve performance [09]. Data processing can also be conducted remotely. Hence, it is more efficient in terms of cost than sending someone to go to monitoring points [10]. Wireless sensor technology can be built with an independent network free from certain network providers. Some early warning systems have been implemented for natural disaster warnings such as soil deformation, water quality, earthquake, tsunami, and flood [11-15].

Real-time micro blogging is a data source that can be implemented in the early warning system named micro blogging-monitoring system. Data input can be used for a decision support system using the knowledge-based framework. Combination of artificial intelligence techniques from high and low levels system analyses creates a system that is flexible and sustainable [16].

The rule-based method can also be implemented in this system. Entity identification can be carried out based on the already implemented knowledge-based framework to help categorize messages. This final system has high information accuracy [17]. This research proposes a method of measurement for $\mathrm{CO}$ concentration with a combination of wireless sensor system and a rule-based system of artificial intelligent algorithm. 


\section{RELATED WORKS}

The rule-based algorithm method proposed here works online and in real-time in the instrument built to measure $\mathrm{CO}$ concentration. There are already a number of $\mathrm{CO}$ concentration measurement methods and they rely on the sources measured. Measurement of $\mathrm{CO}$ concentration from liquid sources has been carried to measure Octane Number (ON) because $\mathrm{CO}$ concentration also relates to $\mathrm{ON}[18]$.

Quick measurement of $\mathrm{CO}$ concentration in the atmosphere is essential as this gas can cause death. A sensor that can swiftly response to the effects of $\mathrm{CO}$ has successfully been developed using Eu-doped Ceria film [19]. The use of laser as a sensor for $\mathrm{CO}$ has also been developed to reduce measurement noise and this system is capable of measuring $\mathrm{CO}$ concentration to as small as 2.4 ppm [20]. Concentration of $\mathrm{CO}$ can also be measured using spectroscopy method. This kind of system requires electronic instruments, source sampling method, and certain temperatures and pressures [21].

Measurement of $\mathrm{CO}$ concentration in area has also been performed using satellite imaging. A number of algorithms have successfully correlate satellite image parameters and $\mathrm{CO}$ concentration in wide areas. These algorithms are also capable of finding the cause of widespread $\mathrm{CO}$ pollution in an area [22]. Satellite imaging method has also been combined with simulation method and this results in higher accuracy with the same pattern of global distribution. This combined system has successfully monitor changes in $\mathrm{CO}$ concentrations due to biomass burning, traffic congestion, and industrial activities [23].

The expert system algorithm embedded into the cloud network can also be used to record events for data analyses and speedy warning via the Internet to any users requiring such information [24]. The use of rulebased for decision support system in the wireless sensor network has also been implemented for water source monitoring systems. This system is capable of monitoring chemical substances in water. The rulebased method in this system reduces computing load for large-scale systems [25]. Combining rule-based method with other systems like fuzzy, can also improve accurate prediction and decision-making speed [26].

Rule-based is part of the artificial intelligence, in which a system manipulates input in the cloud in order to provide speedy and automatic information [27]. Mathematically, the rule-based system is stated in the following equations (1), a combination of rules $r\left(m_{1}, m_{2} \ldots \ldots . . m_{n}\right)$ can be written as equations (2) [28]. A reasoning process is rerun prior to another reasoning process even though the earlier one has finished [29]. In general, rules state what must and must not be done depending on the situation. Rules have a typical format:

If $<$ conditions $>$ then $<$ conclusion

$$
\begin{aligned}
& p \Rightarrow \text { if }(x)^{(1)} \text { is } \bar{F}_{(\rho)}^{(1)} \text { and/or .... and/or is } \bar{F}_{(\rho)}^{(n)} \text { then } y \text { is } \bar{C}_{(\rho)} \\
& r\left(m_{1}, m_{2} \ldots \ldots m_{n}\right)=\left\{\begin{array}{l}
\text { if }(x)^{(1)} i s \bar{F}_{1}^{(1)} \text { and/or....and/or } x^{(n)} i s \bar{F}_{(1)}^{(n)} \text { then } y \text { is } \bar{C}_{\{1 \ldots 1\}} \\
i f(x)^{(1)} i s \bar{F}_{M_{1}}{ }^{(1)} \text { and /or....and/or } x^{(n)} i s \bar{F}_{M_{n}}^{(n)} \text { then } y \text { is } \bar{C}_{\left\{M_{1} . M_{n}\right\}}
\end{array}\right.
\end{aligned}
$$

This research aims to build an information system in the form of a decision support system for early warning of $\mathrm{CO}$ threat that is capable of automatic notification via cellular phones. This system is expected to provide speedy information on imminent $\mathrm{CO}$ threat for proper anticipation. It employs a wireless sensor system to tackle geo-distribution issues [30], and to synchronize decision making elements for speedy decision making [31].

The sensor node functions to conduct data acquisition of carbon monoxide concentration and send those data via the internet. This device continuously sends data to a website using broadband internet access provided by a WIFI-GSM modem to the web server database for storage and management [32]. The use of information system for early warning system in a location can anticipate emergency events that might otherwise be destructive [33].

\section{REALIZATION OF WIRELESS SENSOR SYSTEM}

In this research, the system build consists of instruments known as a node. The sensor node circuit consists of a $\mathrm{CO}$ sensor and a data acquisition system using WIFI network. The $\mathrm{CO}$ gas sensor node has been built and acquired using an Analogue to Digital Converter (ADC) and has sent data to the cloud network via the Internet with a System-on-Chip (SOC) WIFI microcontroller. The sensor system was calibrated by comparing it with a standard instrument available. A diagram of the sensor node is shown in Figure 1. 


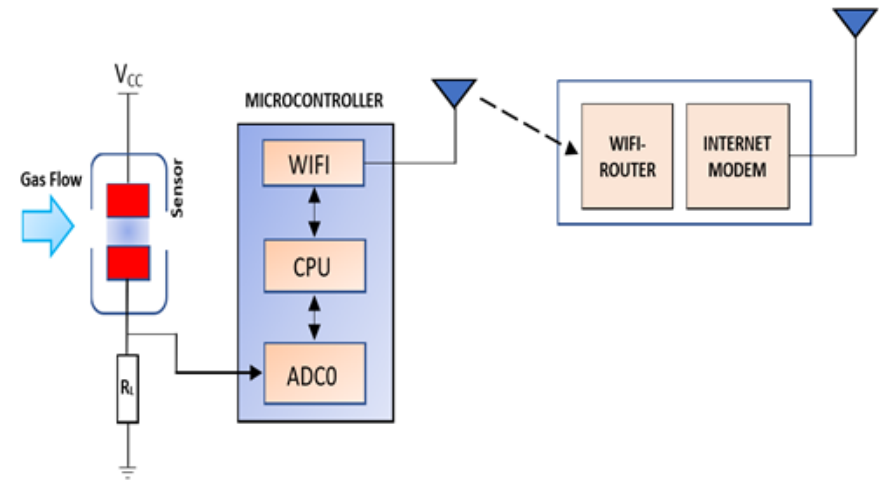

Figure 1: Diagram of CO sensor node connected to the internet

The $\mathrm{CO}$ sensor is made of semiconductor material with low electrical conductivity in clean air. Whenever $\mathrm{CO}$ is around the semiconductor, the absorbed gas molecules result in increasing electrical conductivity in line with $\mathrm{CO}$ concentration. Within the circuit, the sensor output voltage is analogue and is converted into digital data by the ADC0 on the ESP8266 microcontroller.
Measurement results are then sent to the cloud network via an internal WIFI chip in the microcontroller, which is connected to a WIFI router and an Internet modem. Efficacy of the system developed was tested by comparing concentration detected next to the sensor node and the one read on the cloud network. An overview of the network system is shown in Figure 2.

A rule-based expert system programming algorithm is developed in a web host to make decisions on the conditions of carbon monoxide concentration. This system performs two computations using the rule-based algorithm. First, rule-based forward chaining to determine categories of pollution levels and rule-based expert system to determine conditions to automatically trigger the early warning system. The rule-based expert system uses danger status set earlier by environmental and/or disaster experts. Output of the rule-based system is data that are accessible with computers or are warnings sent to mobile phones.
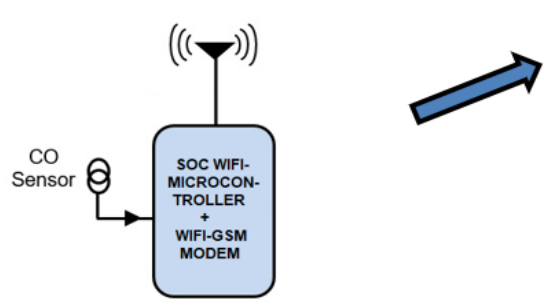

Station-1
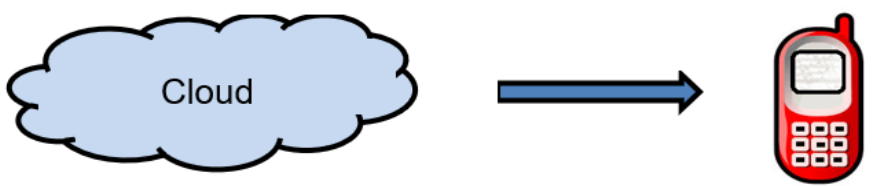

Mobile phone
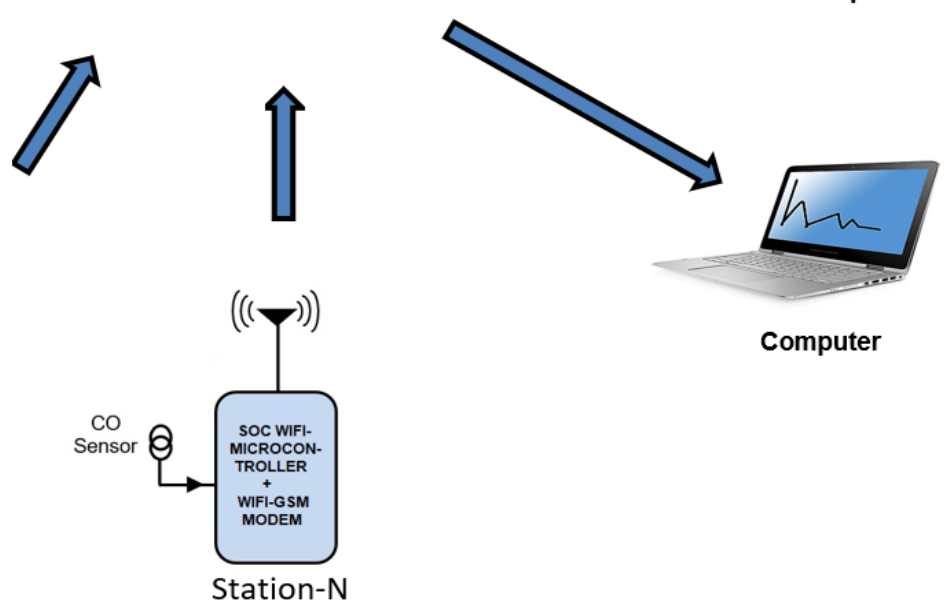

Computer

Figure 2: Carbon monoxide network for the online system

\section{RULE-BASED SYSTEM DEVELOPMENT}

Sensor input of the rule-based system is sent by the sensor node via the Internet. Data base of the rule-based system is a set of rules provided by the administrator and is saved in the cloud data base. The architecture of the rule-based system in this research is shown in Figure 3. The system here has two rule bases; first, from the Indonesian Air Pollutant Index (IAPI), which is used to classify pollution level and, second, from the experts, which is used to determine the emergency status.

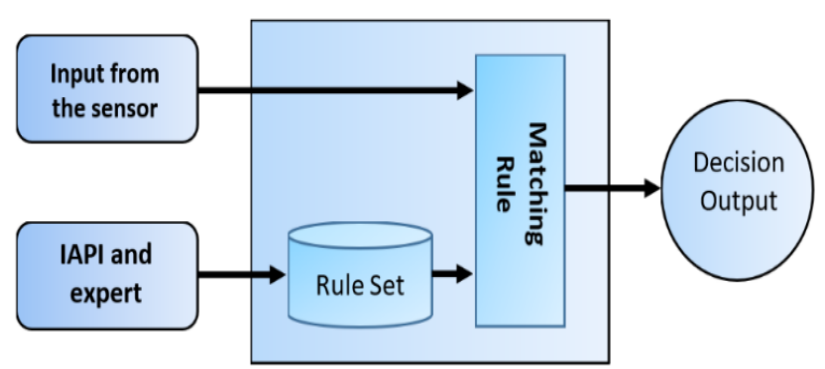

Figure 3: Architecture of the rule-based system 


\section{Rule-based Reasoning System}

The rule-based reasoning in this system is an algorithm to solve problems that use knowledge from an application domain in the form of rules. Rule-based reasoning technique uses a strategy of conflict resolution by choosing one rule from a set of rules. This chosen rule is applied to find the right solution to a problem. Contents of the working memory are then subsequently updated based on that solution. Search for rules ensues as the working memory is kept on updated, and the reasoning process proceeds based on new matching rules. This process continues until all required solutions are obtained and there are no more rules left that fit the ones in the working memory.
Backward chaining is the same as forward chaining in most of the processes. The main difference is in taking problem descriptions as a group of conclusions from a condition, and trying to find premises based on those conclusions. This means finding rules that fit with all or most of the conclusions in the working memory. On the other hand, forward chaining is a conflict resolving strategy by choosing one rule from one set of applicable rules. The Indonesian Air Pollutant Index (IAPI) is a set of data used to simplify information on air pollution agents in which $\mathrm{CO}$ is also listed. The values in the IAPI categories are based on the Decree of the State Minister for the Environment No. KEP-45/MENLH/I0/1997. The rules set in the IAPI category are shown in Table 1.

Table 1: Rules used in the reasoning system

\begin{tabular}{|c|c|}
\hline Rule & Statement \\
\hline 1 & IF (CO input concentration $\leq 50 \mu \mathrm{g} / \mathrm{m}^{3}$ ) THEN Category is Good \\
\hline 2 & IF (CO input concentration $51 \mu \mathrm{g} / \mathrm{m}^{3}$ to $100 \mu \mathrm{g} / \mathrm{m}^{3}$ ) THEN Category is Medium \\
\hline 3 & IF (CO input concentration $101 \mu \mathrm{g} / \mathrm{m}^{3}$ to $199 \mu \mathrm{g} / \mathrm{m}^{3}$ ) THEN Category is Unhealthy \\
\hline 4 & IF (CO input concentration $200 \mu \mathrm{\mu g} / \mathrm{m}^{3}$ to $299 \mathrm{\mu g} / \mathrm{m}^{3}$ ) THEN Category is Very Unhealthy \\
\hline 5 & IF (CO input concentration $\geq 300 \mu \mathrm{g} / \mathrm{m}^{3}$ ) THEN Category is Dangerous \\
\hline
\end{tabular}

\section{Rule-Based Expert System}

In order to meet the condition of an early warning system, rules from environmental experts will also be used rules from those experts are put into the knowledge-based system in the host website. This part requires informed decision from environmental experts to be used as knowledge for the rule-based system. These experts are from the State Ministry for the Environment, with expertise in disaster management. These knowledge-based rules on the early warning of CO pollution include:

1. If there are two detections of a value of $\geq 300$ of IAPI within 3 measurements, the system will automatically give a warning alert.

2. If the IAPI value is $\geq 101$, the system will automatically give a warning alert.

3. If the IAPI values is $<101$, will not give or will stop giving a warning alert.

4. A warning alert is a repeated alarm every 30 minutes until the IAPI value is $<101$

\section{RESULT AND DISCUSSION}

Results of application of online and real-time rule-based system for environmental carbon monoxide monitoring using wireless sensor system can be accessed via the internet. The instrument system developed in this research is shown in Figure 4. Data that can be accessed from the internet include: map of sensor location, acquisition time, concentration value, and status of air pollution index. Measurement results of carbon monoxide concentration are kept in the database of a web server and are shown in real-time as graphs. An expert system programming based on expert knowledge is then used to make decision on pollution.

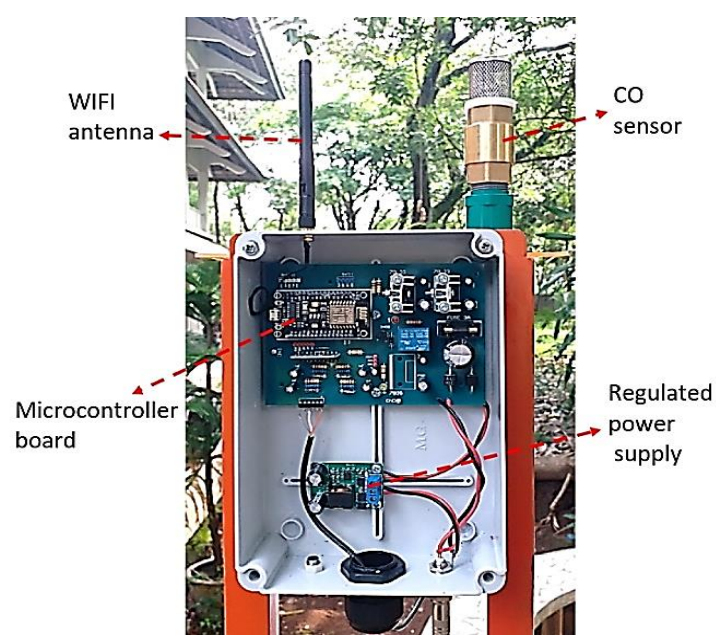

Figure 4: The $\mathrm{CO}$ concentration measurement instrument system developed

system was calibrated against a standard instrument in the measurement range of $0-440 \mu \mathrm{g} / \mathrm{m}^{2}$, as shown in Figure 5. Testing results show that linear correlation of the instrument built against standard measurement instrument is 0.9994 (almost 1). This value means that the instrument built has close proximity to the available manual measurement instrument used as reference. Efficacy of the measurement system is then calculated mathematically using the Root Mean Square Error (RMSE) and relative error formula [34]:

$$
\begin{aligned}
& \text { RMSE }=\sqrt{\frac{\sum_{i=1}^{n}\left(P_{i}-O_{i}\right)}{n}} \\
& \mathrm{E}_{\mathrm{P}}=\left|\left(\mathrm{P}_{\mathrm{i}}-\mathrm{O}_{\mathrm{i}}\right) / \mathrm{O}_{\mathrm{i}}\right| \times 100 \%
\end{aligned}
$$


Where $\mathrm{P}_{\mathrm{i}}$ is gas concentration on the $\mathrm{i}$-th measurement, $\mathrm{O}_{i}$ is $\mathrm{CO}$ concentration on the $\mathrm{i}$-th measurement using standard instrument, and $\mathrm{n}$ is the number of measured data. This research results in RMSE $=3.46 \mu \mathrm{g} / \mathrm{m}^{2}$, in the value range of $0-440 \mu \mathrm{g} / \mathrm{m}^{2}$, with a relative error $\left(E_{p}\right)$ of $0.78 \%$.

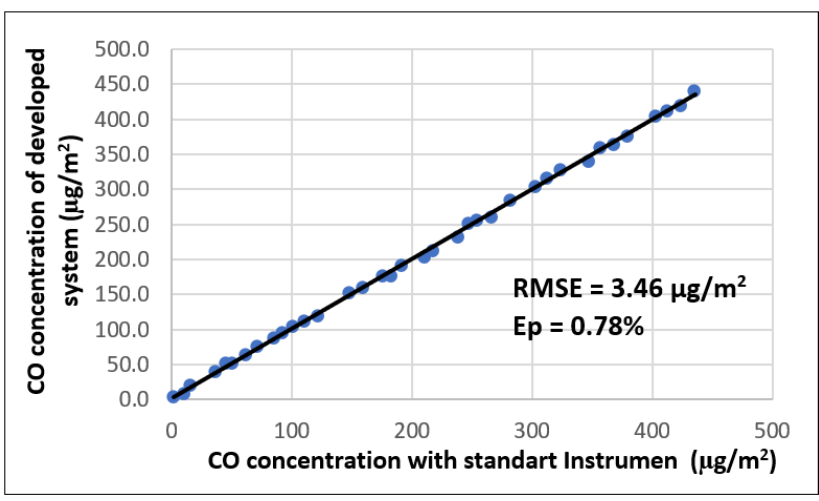

Figure 5: Calibration graph of the sensor node and a standard instrument

Figure 6 depicts results of $\mathrm{CO}$ measurements from 5 sensor nodes in real-time. The graph in Figure 3 shows changes in $\mathrm{CO}$ concentration with time. Values from online and real-time $\mathrm{CO}$ concentration monitoring are displayed in numbers, fluctuating graph, and indicator panel. The graph is a serial display set to show acquisition results from three (3) days of observation. Nonetheless, acquired data from each sensor are stored continuously and are kept in the server over time (day by day). It can be seen in the graph that sensor reading of $\mathrm{CO}$ concentration fluctuates. These values significantly change in the morning, at noon, in the afternoon, in the evening, at night, and in the early morning.

Analyses show that $\mathrm{CO}$ concentration during the day is higher compared to during the night. During the night, from 10:00 pm onwards, CO concentration lowers drastically and reaches its lowest point in the early morning (02:00 am - 03:00 am). Literature studies also indicate that $\mathrm{CO}$ generally comes from vehicle exhausts, biomass burning, and industrial activities [23]. Meanwhile sensor analyses indicate that $\mathrm{CO}$ concentrations measured in this research stem from sources in urban areas, but not from industrial activities or biomass burning. Therefore, it is certain that fluctuating $\mathrm{CO}$ concentrations are caused by vehicle exhausts that are the main transportation means of the area.

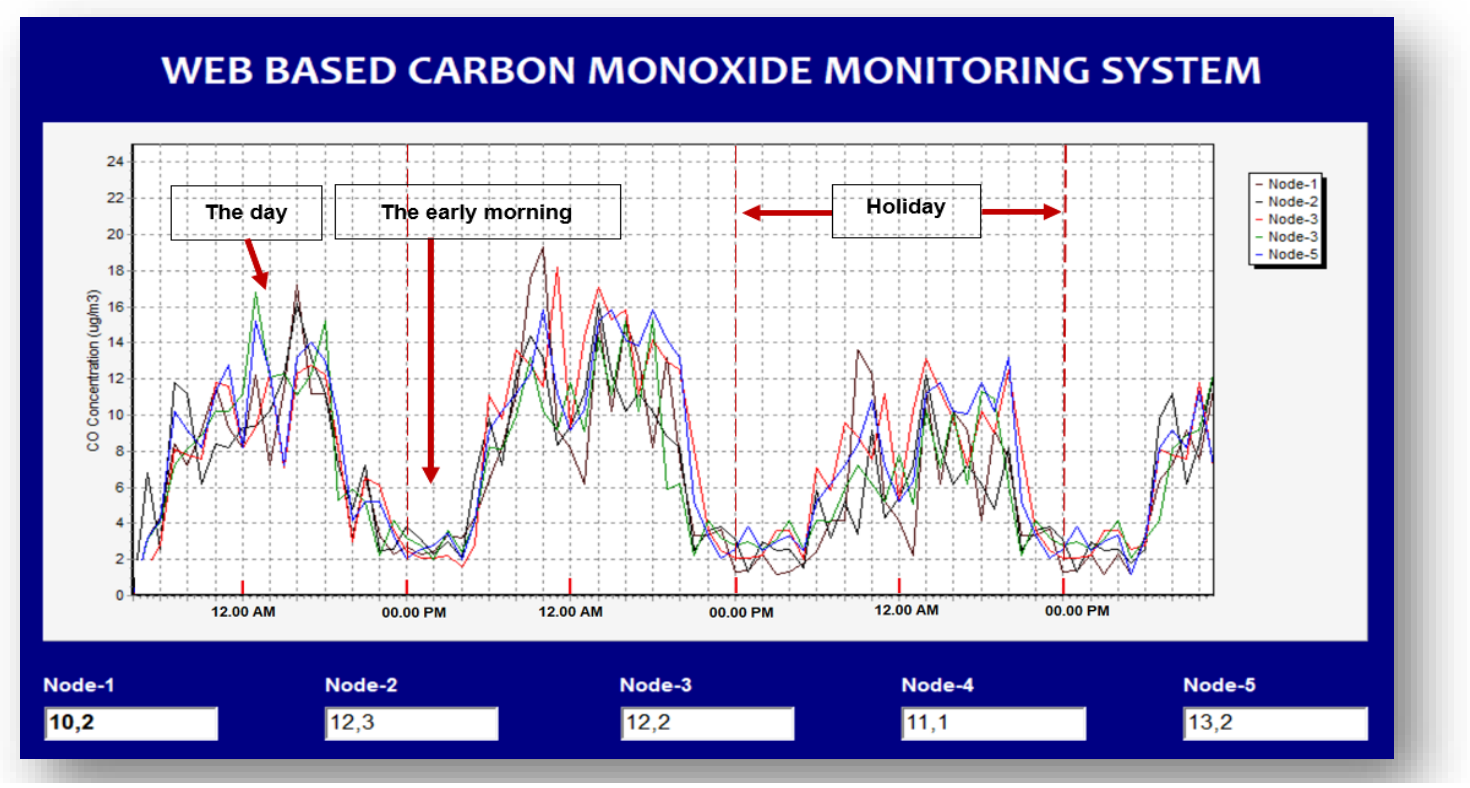

Figure 6: CO measurement at sensor node as displayed in real-time

Further analyses classify mean concentration from $\mathrm{CO}$ readings on a daily basis. A data for one (1) month was used as the results are further categorized, as shown in Figure 7. It can be seen in Figure 7 that $\mathrm{CO}$ concentration is lowest on Sundays when there is low traffic and $\mathrm{CO}$ concentration is highest on Fridays when traffic is at its peak. This finding confirms that the source of $\mathrm{CO}$ concentration in the area is vehicle exhausts.
Data communication system is tested for transmission time between the sensor node and the online web server for every data with ID codes. This dashboard displays calculations that meet the IAPI requirement using the following formula:

$$
I=\frac{I_{A}-I_{S}}{X_{A}-X_{S}}\left(X_{X}-X_{B}\right)+I_{B}
$$




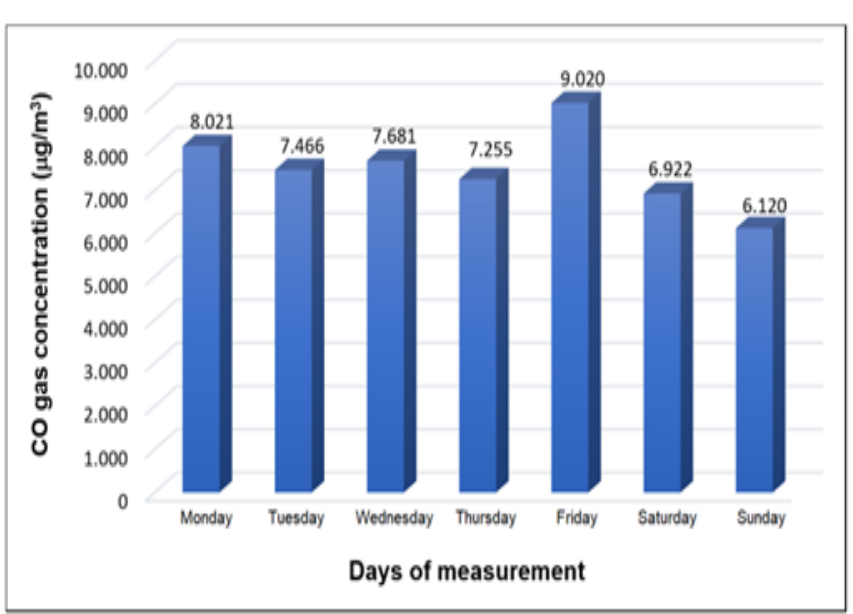

Figure 7: Results of CO concentration measurement by day

Where I is computed IAPI, $I_{A}$ is the Standard Index of Air Pollution (ISPU) upper limit, $I_{B}$ is the ISPU lower limit, $X_{A}$ is the ambient upper limit, $X_{B}$ is the ambient lower limit, and $X_{x}$ is the ambient measurement result. Results of calculations that adhere to the IAPI rule do not only yield index numbers but also statements of conditions and reporting colors in line with the IAPI. Calculation results are not only used to make statements of conditions and reporting displays but also to model rule number 2 that will be used for the early warning of detrimental $\mathrm{CO}$ concentration. This warning is based on the rules set by experts.

Those results show that a warning is not only based on calculations resulting from IAPI rules, but also from expert statements written in the knowledge-based system. This system reveals that certain categories of IAPI may result in different warning conditions. This is due to the effect of knowledge-based system set by experts that requires three readings of the same IAPI value to be worthy of a warning. This research performed validation for the rule-based system by manually recording measurements and comparing them with calculations made by the system built. Validation covered values from low to high concentration of $\mathrm{CO}$ that it properly represented measurements carried out by the rule-based system. Results of this validation process are given in Table 2.

These results were compared to those from standard (manual) measurements. It was subsequently confirmed that both rule-based calculation and manual measurements yield similar results for various ranges of carbon monoxide concentration. Hence, the system can be used. Testing for data transfer rate (throughput) from sensor node to web server has been conducted to figure out data communication effectiveness. This is very important as it affects system response for data reception, rule-based execution, and warning of measured $\mathrm{CO}$ concentration. Testing results for each node are given in Figure 8a.

They show that on average, data transfer rate from sensor node to web server is in 764 milliseconds. It can also be seen that the highest transfer rate is between mid-night and early morning, whereas the highest delay time is experienced in the afternoon. Each sensor node comes with different data transfer rate performance, which is also influenced by GSM network bandwidth, signal strength, and network traffic, among others [35].

Table 2 : Results of rule-based system validation against standard measurement

\begin{tabular}{|l|c|c|l|c|c|}
\hline Node ID & $\begin{array}{c}\text { Developed system } \\
\text { measurement } \\
\left(\mu \mathrm{g} / \mathrm{m}^{3}\right)\end{array}$ & $\begin{array}{c}\text { IAPI } \\
(\mu \mathrm{g} / \mathrm{m} 3)\end{array}$ & $\begin{array}{c}\text { Category } \\
(\text { Rule based system })\end{array}$ & $\begin{array}{c}\text { Reference } \\
\text { measurement } \\
\left(\mu \mathrm{g} / \mathrm{m}^{3}\right)\end{array}$ & $\begin{array}{c}\text { Validation } \\
\text { Statement }\end{array}$ \\
\hline Node-1 & 4.27 & 44 & Good health effect & 4.35 & True \\
\hline Node-2 & 8.10 & 79 & Medium health effect & 7.85 & True \\
\hline Node-3 & 19.85 & 217 & Very unhealthy effect & 20.10 & True \\
\hline Node-4 & 36.60 & 322 & Dangerous healthy effect & 37.01 & True \\
\hline Node-5 & 10.95 & 114 & Unhealthy effect & 10.23 & True \\
\hline Node-1 & 6.20 & 62 & Medium health effect & 6.35 & True \\
\hline Node-2 & 5.35 & 54 & Medium health effect & 5.15 & True \\
\hline Node-3 & 1.60 & 16 & Good health effect & 1.22 & True \\
\hline Node-4 & 49.35 & 429 & Dangerous healthy effect & 47.75 & True \\
\hline Node-5 & 20.00 & 218 & Very unhealthy effect & 19.52 & True \\
\hline
\end{tabular}

Testing for warning time was performed by giving markers from the time data are acquired by the WIFImicrocontroller to the moment warnings are received by cellular phone. This testing also took consideration of network traffic of the Internet and the cellular phones. Testing results for each sensor node are given in Figure
$8 b$. It can be seen that the average warning rate for all networks is 15.6 second, which is still very effective for early warning of carbon monoxide threat. As in the case for data transfer rate testing, differences in sensor node performance are affected by GSM network bandwidth, signal strength, and network traffic. 


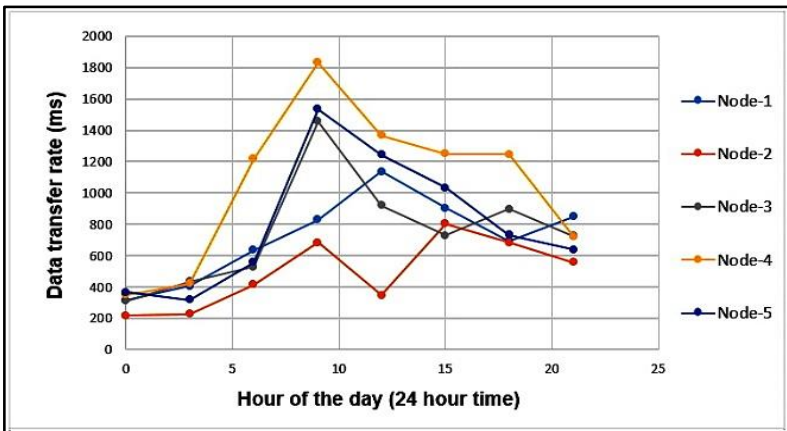

(a)

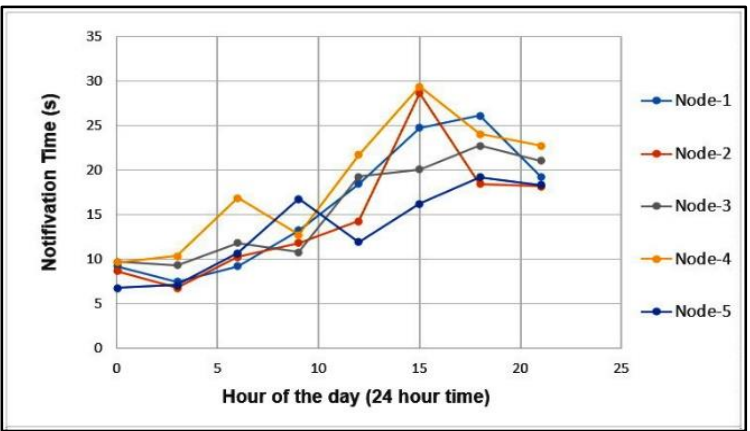

(b)

Figure 8: Testing results for (a) data transfer rate from sensor nodes to the web server and (b) notification response time from sensor nodes to cellular phones

\section{CONCLUSIONS}

The online web-based rule-based system has been proven to be effective and speedy in making decision concerning $\mathrm{CO}$ concentration in the environment using data from wireless node sensor. The use of online database system for $\mathrm{CO}$ concentration measurement is capable of periodic monitoring with values that can directly be linked to the pollution source. The rule-based system allows such data to be managed in line with the regulation observed in a country and the results to be used in determining categories of air pollution. Rulebased system to evaluate $\mathrm{CO}$ concentration can also be combined with a knowledge-based system set by environmental experts. Testing of the network system built shows data transfer speed from sensor node to the server. Meanwhile, testing of user notification by the wireless sensor system reveals a speedy $\mathrm{CO}$ warning. This system contributes to $\mathrm{CO}$ concentration monitoring and hence, helps to improve quality of the environment and health of the people. Measurement data are kept in the database history for a very long time. This means that they can also be used in big data analysis for monitoring of environmental changes.

\section{REFERENCES}

1. Mir, M.A., Bhat, M.A., Majid, S. A., Lone, S.H., Malla, M.A., Tiwari, K.R., Pandit, A.H., Tomar, R., Bhat, T.A. (2018). Studies on the synthesis and characterization of polyaniline zeolite nanostructures and their role in carbon monoxide sensing. Environmental Chemical Engineering, vol. 6, pp.1137-1146.

2. Aggarwal, P., Jain, S. (2015). Impact of air pollutants from surface transport sources on human health: A modeling and epidemiological approach. Environment International, vol. 83, pp.146-157.

3. Levy, R.J. (2015). Carbon monoxide pollution and neurodevelopment: a public health concern. Neurotoxicol. Teratol, vol. 49, pp. 31-40.

4. Sandilands, E.A., Bateman, D.N. (2016). Carbon monoxide. Medicine. Vol. 44, Issue 3, pp. 151-152.
5. Dingenena, J. V., Steiger, C., Zehe, M., Meinel, L., Lefebvre, R.A. (2018). Investigation of orally delivered carbon monoxide for postoperative ileus. European Journal of Pharmaceutics and Biopharmaceutics, vol. 130, pp. 306-313.

6. Somov, A., Baranov, A., Savkin, A., Spirjakin, D., Spirjakin, A., Passerone, R. (2011). Development of wireless sensor network for combustible gas monitoring. Sensors and Actuators A: Physical, vol. 171 , pp. 398- 405.

7. Li, H., Lin, Z. (2017). Study on location of wireless sensor network node in forest environment. Procedia Computer Science, vol. 107, pp. 697-704.

8. Wu, M., Tan, L., Xiong, N. (2016). Data prediction, compression, and recovery in clustered wireless sensor networks for environmental monitoring applications. Information Sciences, vol. 329, pp. 800-818.

9. Denov, A., Milochkin, A. (2016). Wireless data transfer channel in the monitoring systems of oil production wells. Journal of Applied Engineering Science, vol. 14, pp. $477-480$.

10. Vujic, D. (2015). Wireless sensor networks applications in aircraft structural health monitoring. Journal of Applied Engineering Science, vol. 13, pp. 79-86.

11. Kuang, K. S. C. (2018). Wireless chemiluminescence-based sensor for soil deformation detection. Sensors and Actuators A: Physical, vol. 269, pp. 70-78.

12. Jiang, Y., Yang, X., Liang, P., Liu, P., Huang, X. (2018). Microbial fuel cell sensors for water quality early warning systems: fundamentals, signal resolution, optimization and future challenges. Renewable and Sustainable Energy Reviews, vol. 81, pp. 292-305.

13. Zambrano, A. M., Perez, I., Palau, C., Esteve, M. (2017). Technologies of internet of things applied to an earthquake early warning system. Future Generation Computer Systems, vol. 75, pp. 206215.

14. Ai, F., Comfort, L.K., Dong, Y., Znati, T. (2016). A dynamic decision support system based on geographical information and mobile social 
networks: a model for tsunami risk mitigation in Padang, Indonesia. Safety Science, vol. 90, pp. 6274.

15. Krzhizhanovskaya, V. V., Shirshov, G.S., Melnikova, N.B., Belleman, R.G., Rusadi, F.I., Broekhuijsen, B.J., Gouldby, B.P., Lhomme, J., Balis, B., Bubak, M., Pyayt, A.L., Mokhov, I.I., Ozhigin, A.V., Lang, B., Meijer, R.J. (2011). Flood early warning system: design, implementation and computational modules. Procedia Computer Science 4 : International Conference on Computational Science (ICCS) 2011, pp.106-115.

16. Kumar, S.P.L. (2017). State of the art - intense review on artificial intelligence systems application in process planning and manufacturing. Engineering Applications of Artificial Intelligence, vol. 65 , pp. 294-329.

17. Lukasiewicz, K., Teymourian, K., Paschke, A. (2014). A rule-based system for monitoring of micro-blogging disease report. The Semantic Web: ESWC 2014 Satellite Events, pp. 401-406.

18. Diaz, G. J. A, Martinez, L. M. C., Montoya, J. P. G., Olsen, D. B. (2019). Methane number measurements of hydrogen / carbon monoxide mixtures diluted with carbon dioxide for syngas spark ignited internal combustion engine applications. Fuel, vol. 236, pp. 535-543.

19. Ortega, P. P., Rocha, L.S.R., Cortés, J.A., Ramirez, M.A., Buono, C., Ponce, M.A., Simões, A.Z. (2019). Towards carbon monoxide sensors based on europium doped cerium dioxide. Applied Surface Science, vol. 464, pp. 692-699.

20. Nasir, E. F., Farooq, A. (2018). Intra-pulse cavity enhanced measurements of carbon monoxide in a rapid compression machine, IEEE Xplore : 2018 Conference on Lasers and Electro-Optics (CLEO), pp. 1-2.

21. Borkov, Y.G., Petrova, T.M., Solodov, A.M., Solodov, A.A. (2019). Measurements of the broadening and shift parameters of the carbon monoxide spectral lines in the 1-0 band induced by pressure of carbon dioxide. Journal of Quantitative Spectroscopy and Radiative Transfer, vol. 219, pp. 379-382.

22. Mok, J., Park, S. S., Lim, H. K., Kim, J., Edwards, D. P., Lee, J., Yoon, J., Lee, Y. G., Koo, J. (2017). Correlation analysis between regional carbon monoxide and black carbon from satellite measurements. Atmospheric Research, vol. 196, pp. 29-39.

23. Luo, M., Shephard, M. W., Cady-Pereira, K. E, Henze, D. K., Zhu, L., Bash, J. O, Pinder R. W., Capps, S. L., Walker, J.T., Jones, M. R. (2015). Satellite observations of tropospheric ammonia and carbon monoxide: Global distributions, regional correlations and comparisons to model simulations. Atmospheric Environment, vol. 106, pp. 262-277.

24. Karaca, Y., Moonis, M., Zhang, Y., Gezgez, C. (2018). Mobile cloud computing based stroke healthcare system. International Journal of Information Management, Article in press: Available online 12 October 2018.

25. Chang, N., Pongsanone, N. P., Ernest, A. (2012). A rule-based decision support system for sensor deployment in small drinking water networks. Journal of Cleaner Production, vol. 29, pp. 28-37.

26. Gorzalczany, M.B., Rudzinski, F. (2016). A multiobjective genetic optimization for fast, fuzzy rulebased credit classification with balanced accuracy and interpretability. Applied Soft Computing, vol. 40, pp. 206-220.

27. Minutolo, A., Esposito, M., Pietro, D.G. (2017). Optimization of rule-based systems in mHealth applications. Engineering Applications of Artificial Intelligence 59, pp. 103-121.

28. Gorzalczany, M., Rudzinski, F., (2016). A multiobjective genetic optimization for fast, fuzzy rulebased credit classification with balanced accuracy and interpretability. Applied Soft Computing, vol. 40, pp. 206-220.

29. Moghimi, M., Varjani, A. Y. (2016). New rule-based phishing detection method. Expert Systems with Applications, vol. 53, pp. 231-242.

30. Rahmani, A.M., Gia, T.N., Negash, B., Anzanpour A., Azimi, I., Jiang, M., Liljeberg, P., (2018). Exploiting smart e-Health gateways at the edge of healthcare Internet-of-Things: A fog computing approach. Future Generation Computer Systems 78, pp. 641-658.

31. Ferrada, X., Núñez, D., Neyem, A., Serpell, A., Sepúlveda, M. (2016). A cloud-based mobile system to manage lessons-learned in construction projects. Procedia Engineering 164. Pp. 135-142.

32. Zou, H., Zhou.Y., Yang, J., Spanosa, J.C. (2018). Device-free occupancy detection and crowd counting in smart buildings with Wi-Fi-enabled loT. Energy and Buildings, vol.174, pp. 309-322.

33. Mantoro, T., Suryasa, I. N., Moedjiono, S., Nugroho, M. R. (2016). Automatic early warning for vehicles accidents based on user location. Advance Science Letters, vol. 22, pp. 3065-3070.

34. Dutta, L., Hazarika, A., Bhuyan, M. (2018). Nonlinearity compensation of DIC-based multisensor measurement. Measurement, vol. 126, pp. 13-21.

35. Mao, Q., Hu, F., Kumar, S. (2018). Simulation methodology and performance analysis of network coding based transport protocol in wireless big data networks. Simulation Modeling Practice and Theory, vol. 84, pp. 38-49.

Paper submitted: 12.05.2018

Paper accepted: 27.12.2018.

This is an open access article distributed under the CC BY-NC-ND 4.0 terms and conditions 